\title{
STUDIES ON INVASIVE KERATINOPHILIC DERMATOPHYTES OF HUMAN HAIR
}

\author{
*Brajesh Kumar Jha1, S. Mahadeva Murthy² \\ ${ }^{1}$ Research Scholar, Department of Microbiology, Yubraja College, Mysore, India \\ ${ }^{2}$ Associate Professor, Department of Microbiology, Yubraja College, Mysore, India \\ *Corresponding Author's Email: baiju000@ rediffmail.com Phone: +977- 9845087892
}

\begin{abstract}
:
Background: Tinea Capitis (TC) is a dermatophyte infection of the scalp hair follicles and intervening skin. TC is mainly caused by anthropohilic and zoophilic species of the genera Trichophyton and Microsporum. On the basis of the type of hair invasion, dermatophytes are also classified as endothrix, ectothrix or favus. Despite the availability of effective antifungal agents, dermatophytic infections continue to be one of the principal dermatological diseases in Mysore.

Objectives: To study the genus and species variants, of fungus causing Tinea Capitis infection and epidemiological factors responsible for the disease in Central Mysore.

Materials and methods: Clinically suspected 527 patients with dermatophytes infection cases were included in our study, where 58 cases were diagnosed and confirmed as a Tinea Capitis patients only selected for our study. Suspected lesion like scalp skin scraping and hair plucking samples were collected after disinfecting the site with $70 \%$ of ethyl alcohol. Samples were collected in a sterile thick black envelope, folded, labelled and brought to the laboratory for further processing according to slandered Mycological protocol.

Results: A total of 527 patients with dermatophytes infection suspected cases were included in our study, where 58 cases $(11.0 \%)$ were confirmed as a Tinea Capitis. Majority of the dermatophytic infections were seen between April to October i.e. 51, $63,52,30,51,60$ and 57 respectively, so this signified that hot and humid climate favour for dermatophytic infection. There was 33 cases of endothrix infection and only 12 cases of ectothrix infection and 13 cases were not ascertained but $\mathrm{P}$ value is significant $(\mathrm{P}<0.001)$ Among all dermatophytes Tinea Capitis was mainly caused by only one genus i.e. T. tonsurans $29 / 58$ (34.4\%), T. violaceum 8/58 (31.0\%), T. verrucosum 4/58 (6.8\%) and T. mentagrophytes 8/58 (13.7\%) were isolated.

Conclusion: The prevalence of dermatophyte infections among children was (11.0\%), which is most common among the other clinical type of infection. Poverty, lower socioeconomic status, unhygienic condition, hot humid climate and young age group child is the major factors which intensify the dermatophyte infection as a cause of Tinea Capitis. There for continuous isolation identification and conformation of dermatophyte is essential to treat with antifungal drugs for their complete cure.

Key words: Tinea Capitis, dermatophytes, Trichophyton tonsurans.
\end{abstract}

\section{INTRODUCTION:}

Dermatophytoses are infections of skin, hair, and nails caused by species of the fungal genera Trichophyton, Microsporum, and Epidermophyton. ${ }^{1-3}$ Tinea capitis (TC) is a dermatophyte infection of the scalp hair follicles and intervening skin. ${ }^{4}$ Dermatophytes are keratinophilic fungi which belong to three genera: Trichophyton, Microsporum, and Epidermophyton. On the basis of host preference and natural habitat, they are classified as anthropophilic, zoophilic, and geophilic. ${ }^{5-8} \mathrm{TC}$ is mainly caused by anthropophilic and zoophilic species of the genera Trichophyton and Microsporum. ${ }^{9}$ On the basis of the type of hair invasion, dermatophytes are also classified as endothrix, ectothrix or favus. In endothrix infection the fungus grows completely within the hair shaft, the hyphae are converted to arthroconidia (spores) within the hair while the cuticle surface of the hair remains intact. In ectothrix infection the hyphae destroy the hair cuticle and grow around the exterior of the hair shaft. ${ }^{11}$ TC occurs primarily in prepubertal children aged 3 to 7 years and occasionally in infants and adults. These dermatophytes can be transmitted from person to person and through fomites. ${ }^{12-14}$ The clinical presentation of the disease varies depending on the aetiological agent and type of hair invasion, the level of host resistance and the degree of inflammatory host response. ${ }^{15-18}$ There are three main clinical forms: a) tinea capitis superficialis (noninflammatory form), b) tinea capitis profunda (inflammatory form), and c) tinea capitis favosa (favus) .The noninflammatory form may have a variety of clinical patterns, ranging from diffuse scaling to black dot alopecia. The inflammatory form of tinea capitis may present as agminate folliculitis or kerion Celsi. Favus is a rare type of TC characterized by typical honey-colored, cup-shaped, follicular crusts called scutula. Asymptomatic carriage (AC) seems to be organism specific. Anthopophilic dermatophytes such as $T$. tonsurans, $T$. violaceum, and $M$. audouinii have been associated with high rates of AC. These organisms generally produce mild signs of infection. Asymptomatic carriers at home or school are potentially important sources of disease transmission. ${ }^{19-21}$

\section{MATERIALS AND METHODS:}

Clinically suspected 527 patients with dermatophytes infection cases were included in our study, where 58 cases were diagnosed and confirmed as a Tinea Capitis patients only selected for our study, from January 2010 to January 2011, K. R. Hospital, Mysore. Suspected lesion like scalp skin scraping and hair plucking samples were collected after disinfecting the site with $70 \%$ of ethyl alcohol. Samples were collected in a sterile thick black envelope, 
folded, labelled and brought to the laboratory for further processing.

Inclusion criteria: All patient without taking any antifungal drugs and age below 14 years were included for our study.

Exclusion criteria: Some patients refuse to go for skin scraping and unable to give proper history of antifungal therapy. People, clinically diagnosed with ectoparasites infestation were also excluded from this study.

\section{Specimen collection}

The specimens were collected, in a sufficient amount, from the edge of the infected area, which corresponds to the active zone of the lesion. Any crusts were carefully removed with tweezers and the lesion was disinfected with $70 \%$ alcohol before sampling to remove contaminants such as bacteria. Hair roots and crusts were plucked and

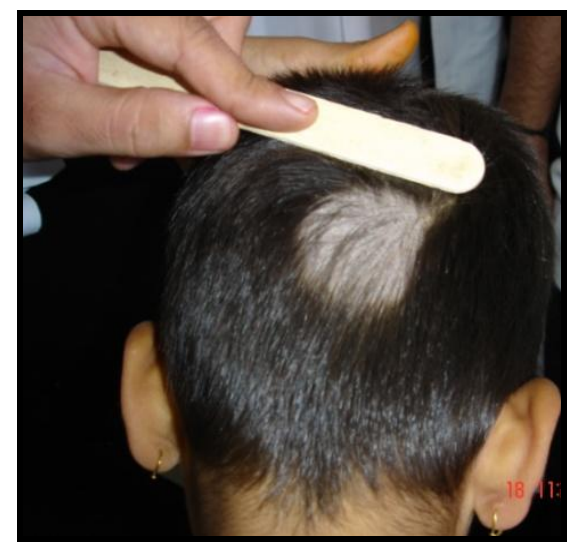

Figure 1: Gray patch type.

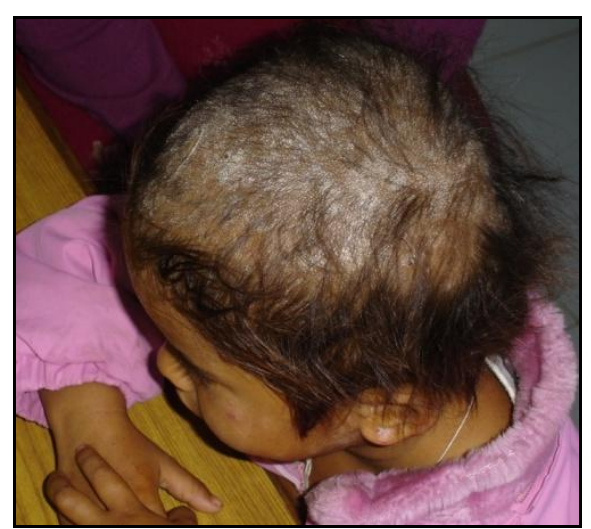

Figure 3: Black dot type

(Diffuse scaling with hair loss)

\section{Microspopic examination}

Direct microscopic examination of skin scrapings and hair is the most rapid method of establishing fungal aetiology. Hair roots and skin scrapings are mounted in 10-20\% potassium hydroxide $(\mathrm{KOH})$ solution with dimethyl sulfoxide (DMSO). The slide was gently heated and viewed under the light microscope. The appearance of infected hairs depends on the invading dermatophyte species so careful observation was done to differentiate suppurating lesions swabbed as seen in Figure-1, 2, 3 and 4. Due to electrostatic attraction, plastic boxes are unsuitable; so that specimens have to be collected in sterile glass containers. ${ }^{22}$ Human asymptomatic carriers may be detected by rubbing the whole scalp or hair with a sterile piece of carpet, a sterile swab humidified with distilled water, a toothbrush or hairbrush and then impressed on the surface of the culture medium. ${ }^{23}$

\section{TINEA CAPITIS:}

\section{Legends for figure:}

Figure-1: Gray patch type.

Figure-2: Inflammatory type (follicular pustules, scaling with hair loss).

Figure-3: Black dot type (diffuse scaling with hair loss).

Figure-4: Kerion type (inflammatory boggy swelling).

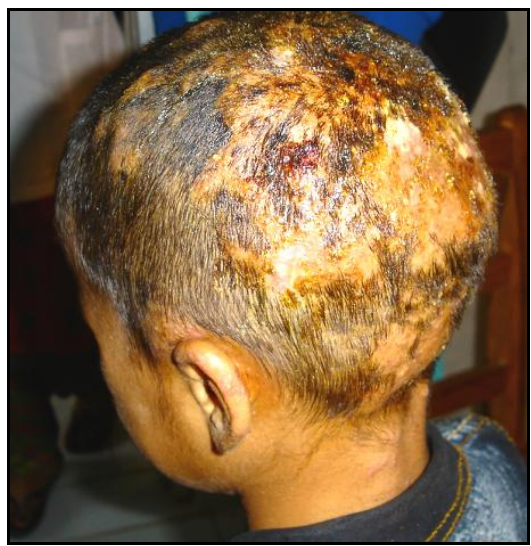

Figure 2: Inflammatory type

(Follicular pustules, scaling with hair loss)

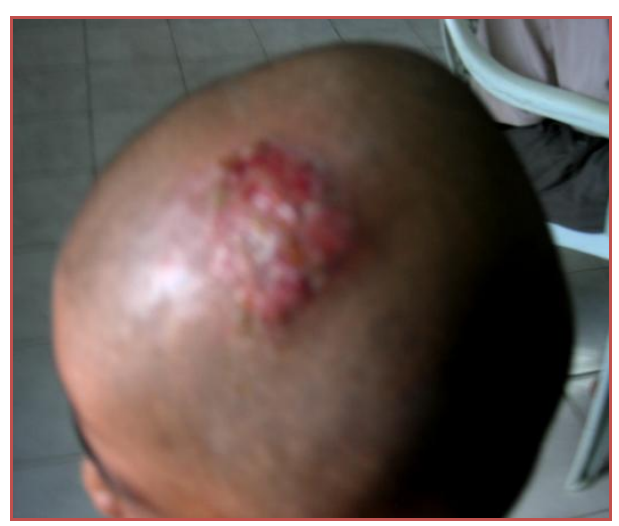

Figure 4: Kerion type

( inflammatory boggy swelling)

from fibres of cotton wool or synthetic fabrics and from "mosaic" which was a network of debris including cholesterol crystals around epidermal cells.

\section{Culture}

Plucked hair fragments and skin scrapings were placed directly on culture medium. The most commonly used medium was Sabouraud's agar with chloramphenicol and cycloheximide which inhibits bacterial and saprobic fungal 
contamination. Cultures were incubated at $20-25^{\circ} \mathrm{C}$ for $3-$ 4 weeks (or for up to 6 weeks if $T$. verrucosum, $T$. violaceum or $T$. sudanense were suspected) and macroscopically screened twice a week for signs of fungal growth. Fungal identification was done based on macroscopic (growth characteristics, pigment formation) as well as microscopic morphology (formation of macroconidia and microconidia or other typical elements). Additionally, in case of atypical isolates, some biochemical or physiological tests were performed such as the search for urease activity or the in vitro hair perforation test. $^{24}$

\section{Wood's light examination}

Wood's light examination of patients hair was also done, to differentiate whether the dermatophyte was an ectothrix or endothrix. Ectothrix dermatophytes such as $M$. canis, $M$. Audouinii and $M$. distortum produced infected hair fluoresce bright green. ${ }^{25}$ On the other hand, endothrix dermatophytes like $T$. tonsurans and $T$. violaceum did not fluoresce at all Wood's light examination was also supportive tool for surveys in ecthotrix anthropohilic cases. $^{26}$

\section{RESULTS:}

The study was a descriptive study on the prevalence of Tinea Capitis caused by dermatophytes in central part of Mysore. A total of 527 patients with dermatophytes infection suspected cases were included in our study, where 58 cases $(11.0 \%)$ were confirmed as a Tinea Capitis. In central Mysore Tinea Capitis is second most common dermatophytic infection after Tinea Corporis. Majority of the dermatophytic infections were seen between April to October i.e. 51, 63, 52, 30, 51, 60 and 57 respectively, so this signified that hot and humid climate favour for dermatophytic infection. Most important thing is that there was no any patient showed evidence of other clinical type of infection among Tinea Capitis patients as shown in Table-1. All 58 cases were screened with wood lamp, only 5 cases showed fluorescence, where as other cases did not show.

Genus and species of dermatophyte were isolated based on morphotyping, biotyping and some required physiological tests to conform as positive culture for dermatophytes, which was out of 527 cases $58(11.0 \%)$ positive only for Tinea Capitis. There was 33 cases of endothrix infection and only 12 cases of ectothrix infection and 13 cases were not ascertained but $\mathrm{P}$ value is significant $(\mathrm{P}<0.001)$ Among all dermatophytes Tinea Capitis was mainly caused by only one genus i.e. T. tonsurans $29 / 58$ (34.4\%), T. violaceum $8 / 58(31.0 \%)$, T. verrucosum $4 / 58(6.8 \%)$ and T. mentagrophytes $8 / 58(13.7 \%)$ were isolated as shown in Table-2.

Table 1: Demographic and socio-economic characteristics of study participants

\begin{tabular}{|c|c|c|c|c|}
\hline Factors & Number of cases & Number of Positive cases & Percentage $\%$ & P-value \\
\hline \multicolumn{5}{|c|}{ 1. Seasonal variation of Tinea Capitis. } \\
\hline Aug. 10 & 51 & 3 & 5.1 & \multirow{13}{*}{$\mathrm{P}<0.2$} \\
\hline Sep. 10 & 60 & 5 & 8.8 & \\
\hline Oct. 10 & 57 & 4 & 6.9 & \\
\hline Nov. 10 & 47 & 6 & 10.3 & \\
\hline Dec. 10 & 38 & 9 & 15.5 & \\
\hline Jan. 11 & 20 & 1 & 1.7 & \\
\hline Feb. 11 & 28 & 7 & 12.1 & \\
\hline Mar. 11 & 30 & 6 & 10.3 & \\
\hline Apr. 11 & 51 & 3 & 5.2 & \\
\hline May. 11 & 63 & 4 & 6.8 & \\
\hline Jun.11 & 52 & 6 & 10.3 & \\
\hline Jul. 11 & 30 & 4 & 6.8 & \\
\hline Total & 527 & 58 & 100 & \\
\hline \multicolumn{5}{|c|}{ 2. Age distribution. } \\
\hline$<1$ & 60 & 8 & 13.8 & \multirow{4}{*}{$\mathrm{P}<0.05$} \\
\hline $1-5$ & 108 & 11 & 18.9 & \\
\hline $6-10$ & 279 & 23 & 39.6 & \\
\hline $11-14$ & 80 & 16 & 27.6 & \\
\hline \multicolumn{5}{|c|}{ 3. Sex distribution. } \\
\hline Male & 356 & 39 & 67.2 & \multirow[b]{2}{*}{$\mathrm{P}<0.001$} \\
\hline Female & 171 & 19 & 32.7 & \\
\hline \multicolumn{5}{|l|}{ 4. Occupation. } \\
\hline Farmers & 213 & 19 & 32.7 & \multirow{3}{*}{$\mathrm{P}<0.001$} \\
\hline Students & 189 & 27 & 46.5 & \\
\hline House wife & 125 & 12 & 20.6 & \\
\hline \multicolumn{5}{|c|}{ 5. Socio-economic status. } \\
\hline Lower & 289 & 31 & 53.4 & \\
\hline
\end{tabular}




\begin{tabular}{|c|c|c|c|c|}
\hline \multicolumn{4}{|c|}{ Table 1 continue ................ } & \multirow[t]{3}{*}{$\mathrm{P}<0.001$} \\
\hline Middle & 151 & 19 & 32.7 & \\
\hline Upper & 87 & 8 & 13.7 & \\
\hline \multicolumn{5}{|c|}{ 6. Place of resident } \\
\hline Urban & 323 & 38 & 65.5 & \multirow[b]{2}{*}{$\mathrm{P}<0.001$} \\
\hline Ruler & 207 & 20 & 34.5 & \\
\hline \multicolumn{5}{|c|}{ 7. Patches of hair loss. } \\
\hline Simple & 230 & 26 & 44.8 & \multirow[b]{2}{*}{$\mathrm{P}<0.001$} \\
\hline Solitary & 297 & 32 & 55.2 & \\
\hline \multicolumn{5}{|c|}{ 8. Duration of the diseases. } \\
\hline $1-4$ & 162 & 31 & 53.4 & \multirow{5}{*}{$\mathrm{P}<0.001$} \\
\hline $5-8$ & 98 & 22 & 37.9 & \\
\hline $9-12$ & 81 & 11 & 18.9 & \\
\hline $13-16$ & 120 & 16 & 27.6 & \\
\hline$>16$ weeks & 66 & 20 & 34.5 & \\
\hline \multicolumn{5}{|c|}{ 9. Clinical complains (Symptoms). } \\
\hline Itching & 430 & 49 & 84.5 & \multirow[b]{2}{*}{$\mathrm{P}<0.001$} \\
\hline Pain & 97 & 9 & 15.5 & \\
\hline \multicolumn{5}{|l|}{ 10. Clinical signs. } \\
\hline 1 patch hair loss & 230 & 18 & 31.0 & \multirow{6}{*}{$\mathrm{P}<0.001$} \\
\hline$>1$ patch hair loss & 96 & 13 & 22.4 & \\
\hline Pustules & 44 & 3 & 5.2 & \\
\hline Crusts & 59 & 10 & 17.2 & \\
\hline Scales & 68 & 7 & 12.0 & \\
\hline Lymph involve & 30 & 7 & 12.0 & \\
\hline \multicolumn{5}{|l|}{ 11. Clinical types. } \\
\hline Grey patch & 278 & 36 & 62.0 & \multirow{3}{*}{$\mathrm{P}<0.001$} \\
\hline Black dot & 193 & 18 & 31.0 & \\
\hline Kerion & 56 & 4 & 6.9 & \\
\hline \multicolumn{5}{|c|}{ 12. Direct microscopy. } \\
\hline Indothrix & 332 & 33 & 56.8 & \multirow{3}{*}{$\mathrm{P}<0.001$} \\
\hline Ectothrix & 162 & 12 & 20.7 & \\
\hline Not ascertained & 33 & 13 & 22.4 & \\
\hline
\end{tabular}

Table 2: Shows different Trichophyton genus involved in hair infection.

\begin{tabular}{|c|c|c|c|c|c|}
\hline 13. Culture & Grey patch & Black dot & Kerion & Total & Percent \\
\hline T. tonsurans & 19 & 18 & - & $\mathbf{2 0}$ & 34.5 \\
\hline T. violaceum & 6 & 2 & - & $\mathbf{8}$ & 13.7 \\
\hline T. verrucosum & 10 & 8 & - & $\mathbf{1 8}$ & 31.0 \\
\hline T. mentagrophytes & - & - & 4 & $\mathbf{4}$ & 6.8 \\
\hline T. rubrum & 8 & - & - & $\mathbf{8}$ & 13.7 \\
\hline Total & $\mathbf{4 3}$ & $\mathbf{2 8}$ & $\mathbf{4}$ & $\mathbf{5 8}$ & $\mathbf{1 0 0}$ \\
\hline
\end{tabular}

\section{DISCUSSION:}

This study was undertaken during a period extending from August 2010 to July 2011. During this period 527 cases of suspected dermatophytosis were seen in the department of Dermatology in the K. R. Hospital, Mysore. The study revelled an overall prevalence of Tinea Capitis was 58 $(11.0 \%)$ among the suspected fungal infected patients. Study done by A Rajgopal et al. ${ }^{27}$ quoted the incidence rate of $11.2 \%$ of Tinea Capitis but another study Tinea Capitis constituted only $4.9 \%$ of clinically diagnosed cases of dermatophytosis.

In developed country like UK and USA 5- $20 \%$ of the population are at risk Rook et al. ${ }^{28}$ in our study majority of cases were seen during April to October 29/58 (50.0\%), which was similar type of result when we compare the observation made by Joseph $\mathrm{P}$ and Serum et al. ${ }^{29,30}$ Result was 29/46 (63.0\%). This gives an idea that hot and humid climate is the major factor for dermatophyte growth and cause of Tinea Capitis. Most of the infected children were aged below 14 years had Tinea Capitis but incidence rate was maximum among 6-10 years group 23 cases (39.6\%). This study also showed male 39 (67.2\%) were more infected then female 19 cases $(32.7 \%)$. A similar type of infection rate was also observed by other two investigators Rajgopal et al. 56 and Rook et al. ${ }^{28}$

Mainly urban people with poor socioeconomic status like farmers labour group child acquired more Tinea Capitis than other group. In poor 31 cases $(53.4 \%)$, urban group 38 cases $(65.5 \%)$ and farmers had 27 cases $(46.0 \%)$. Study done by BSN Reddy et al. ${ }^{31}$ majorities of the cases were from poor socioeconomic group 34 cases $(50.0 \%)$, which is similar with our result but they got rural child with more Tinea Capitis 49 
cases $(71.0 \%)$ which is just opposite to our report. In this study mostly patients presented with the complain of itching lesions of 49 cases $(84.5 \%)$ and only 9 cases showed pain, attended the Dermatology clinic within a month after onset 31 $(53.4 \%)$ with the sign of hair loss. On observation done by Dermatologist, found that grey patches were predominant followed by black dot and Kerion in only few cases. Ectothrix infections were 12 cases $(20.6 \%)$ but 13 cases were not ascertained in which they belong to. No comparative data were available to compare.

In the culture and identification out of 527 cases 58 confirmed cases of Tinea Capitis were selectively taken for our study. $T$. tonsurans was the main predominant causative agent for Tinea Capitis, 20 cases $(34.4 \%)$ out of which 12 cases were grey patches and only 8 cases were black dot. Second most common was $T$. verrucosum with 10 cases of grey patch and 8 cases of black dot and $T$. violaceum also infect same clinical type but T. mentagrophytes 4 cases of kerion, T. rubrum 8 cases of grey patches were found. BNS Reddy et al. 57 found in his study out of 56 cases isolated and confirmed as a Tinea Capitis were taken for study, among them $T$. violaceum was more predominant 45 cases $(66.2 \%)$ where 18 were having grey patches, 15 cases with black dot and 12 cases of kerion type. T. mentagrophytes in 2 cases $(2.9 \%)$ of kerion type and T. rubrum 2 cases of grey patches type, so in our observation

\section{REFERENCES:}

1. Gupta AK, Summerbell RC. Tinea capitis. Med Mycol 2000; 38: 25587.

2. Menan EI, Zongo-Bonou O, Rouet F, KiKI-Barro PC, Yavo W, Guessan NF, et al. Tinea capitis in schoolchildren from lvory Coast (western Africa). A 1998-1999 cross-sectional study. Int J Dermatol 2002; 41:204-7.

3. Ilkit M, Demirhindi H. Asymptomatic dermatophyte scalp carriage: laboratory diagnosis, epidemiology and management. Mycopathologia 2008; 165:61-71

4. Razzaq Adel AA, Sultan AO, Basmiah AM, Aftab A, Nabel N. Prevalence of tinea capitis in southern Kuwait. Mycoses 2007; 50: 317-20

5. Romano C, Gianni C, Papini M. Tinea capitis in infants less than 1 year of age. Pediatr Dermatol. 2001; 18:465-68.

6. Pomeranz AJ, Sabnis SS. Tinea capitis: epidemiology, diagnosis and management strategies. Paediatr Drugs 2002; 4:779-83.

7. Hay RJ, Clayton YM, De Silva N, Midgley G, Rossor E. Tinea capitis in south-east London: a new pattern of infection with public health implications. Br J Dermatol 1996; 135: 955-58.

8. Hay RJ, Robles W, Midgley G, Moore MK. European Confederation of Medical Mycology Working Party on Tinea Capitis. Tinea capitis in Europe: new perspective on an old problem. J Eur Acad Dermatol Venereol 2001; 15: 229-33.

9. Ginter-Hanselmayer G, Weger W, Ilkit M, Smolle J. Epidemiology of tinea capitis in Europe: current state and changing patterns. Mycoses. 2007; 50 Suppl 2:6-13.

10. Niczyporuk W, Krajewska-Kułak E, Lukaszuk C. Tinea capitis favosa in Poland. Mycoses. 2004; 47:257-60.

11. Fuller LC, Child FJ, Midgley G, Higgins EM. Diagnosis and management of scalp ringworm. BMJ. 2003; 326: 539-41.

12. Boralevi F, Léauté-Labrèze C, Roul S, Couprie B, Taïeb A. Lupuserythematosus- Like Eruption Induced by Trichophyton mentagrophytes Infection Dermatology 2003; 206:303-6.

13. Robert R, Pihet M. Conventional methods for the diagnosis of dermatophytes. Mycopathologia 2008; 166:295-306.

14. Panasiti V, Borroni RG, Devirgiliis V, Rossi M, Fabbrizio L, Masciangelo $\mathrm{R}$, et al. Comparison of diagnostic methods in the diagnosis of dermatomycoses and onychomycoses. Mycoses. 2006; 49:26-9.
T. tonsurans is more common type responsible to cause Tinea Capitis.

\section{CONCLUSION:}

The prevalence of dermatophyte infections among children was $(11.0 \%)$, which is most common among the other clinical type of the infection. Poverty, lower socioeconomic status, unhygienic condition, hot humid climate and young age group child is the major factors which intensify the dermatophyte infection as a cause of Tinea Capitis. There for continuous isolation identification and conformation of dermatophyte is essential to treat with antifungal drugs for their complete cure.

\section{RECOMMENDATIONS:}

1. Wering of dry inner garments in hot humid season.

2. Daly bathing to maintain personal hygiene.

3. Health education targeting reduction of fungal infection.

4. Health consciousness programme through media.

5. Full course of treatment without any gap or negligence.

\section{ACKNOWLEDGEMENT:}

To K. R. Hospital, Mysore for allowing me to do this research work in laboratory for processing Mycological sample, P. B. Despande for help in sample processing and my wife Mamta Jha for typing manuscript.

15. Liu D, Coloe S, Baird R, Pedersen J. Application of PCR to the identification of dermatophyte fungi. J Med Microbiol. 2000 ; 49:4937.

16. Kac G. Molecular approaches to the study of dermatophytes. Med Mycol 2000; 38:329-36.

17. Macura AB. Dermatophyte infections. Int J Dermatol 1993; 32: $313-$ 23.

18. Kefalidou S, Odia S, Gruseck E, Schmidt T, Ring J, Abeck D. Wood's light in Microsporum canis positive patients. Mycoses 1997; 40: 461-3.

19. Cowen P. Microscopy of skin scrapings for dermatophyte diagnosis. Aust Fam Physician. 1990; 19 (5):685-90.

20. Kamalam A, Thambiah AS. Tinea capitis in a Moslem school. Indian J Med Res 1979; 70: 40-3.

21. Ginter-Hanselmayer G, Weger W, Ilkit M, Smolle J. Epidemiology of tinea capitis in Europe: current state and changing patterns. Mycoses. 2007; 50 Suppl 2:6-13

22. Niczyporuk W, Krajewska-Kułak E, Łukaszuk C. Tinea capitis favosa in Poland. Mycoses. 2004; 47:257-60.

23. Prevost E. The rise and fall of fluorescent tinea capitis. Pediatr Dermatol. 1983 Oct; 1(2):127-33.

24. Honig PJ, Sullivan K, McGowan KL. The rapid diagnosis of tinea capitis using calcofluor white. Pediatr Emerg Care. 1996; 12 (5):333-5.

25. Goldgeier MH. Fungal infections of the skin, hair, and nails. Pediatr Ann. 1993; 22(4):253-9.

26. Hubbard TW, de Triquet JM. Brush-culture method for diagnosing tinea capitis. Pediatrics. 1992 Sep; 90(3):416-8.

27. Rajgopal A, Girgilla HS, Pande SS. Clinico mycological and immunological study of Tinea Capitis. IJDVL 1981; 4:146-52.

28. Rook FB, Cattle B. Ring worm. Br. Med. J 1954; 11:198-200.

29. Joseph BM et al. Tinea Capitis by T.tonsurans. California medicine 1955:83-371.

30. Serum JT, Van Avermaete D, Kelley LM. Analysis of tests used to differentiate Trichophyton rubrum from Trichophyton mentagrophytes. J Clin Microbiol. 1981 Jan; 13(1):62-5.

31. Reddy BNS, Nath G, Garg BR. Clinico mycological study of Tinea Capitis. IJDVL 1991; 5:180-82. 